\title{
Red Cell Distribution Width Is Independently Associated with Mortality in Sepsis
}

\author{
Daniel Dankl ${ }^{\mathrm{a}}$ Richard Rezar ${ }^{\mathrm{b}}$ Behrooz Mamandipoor ${ }^{\mathrm{c}}$ Zhichao Zhou $^{\mathrm{d}}$ \\ Sarah Wernly ${ }^{\mathrm{e}} \mathrm{f}$ Bernhard Wernly ${ }^{\mathrm{e}} \mathrm{f}$ Venet Osmani ${ }^{\mathrm{c}}$ \\ aDepartment of Anaesthesiology, Perioperative Medicine and Intensive Care Medicine, Paracelsus Medical \\ University of Salzburg, Salzburg, Austria; bepartment of Cardiology and Intensive Care Medicine, Paracelsus \\ Medical University of Salzburg, Salzburg, Austria; 'Fondazione Bruno Kessler Research Institute, Trento, Italy; \\ ${ }^{d}$ Division of Cardiology, Department of Medicine, Karolinska Institutet, Karolinska University Hospital, Stockholm, \\ Sweden; ' Center for Public Health and Healthcare Research, Paracelsus Medical University of Salzburg, Salzburg, \\ Austria; ${ }^{\text {DDepartment }}$ of Internal Medicine, General Hospital Oberndorf, Teaching Hospital of the Paracelsus \\ Medical University Salzburg, Oberndorf, Austria
}

\section{Highlights of the Study}

- Red cell distribution width is independently associated with mortality in sepsis.

- Combining red cell distribution width and serum lactate showed similar prognostic performance compared to Sequential Organ Failure Assessment (SOFA) and Acute Physiology and Chronic Health Evaluation (APACHE) IV scores.

\section{Keywords}

Sepsis · Intensive care $\cdot$ Red cell distribution width

\begin{abstract}
Background: Mortality in sepsis remains high. Studies on small cohorts have shown that red cell distribution width (RDW) is associated with mortality. The aim of this study was to validate these findings in a large multicenter cohort. Methods: We conducted this retrospective analysis of the multicenter eICU Collaborative Research Database in 16,423 septic patients. We split the cohort in patients with low ( $\leq 15 \% ; n=7,129)$ and high (>15\%; $n=9,294)$ RDW. Univariable and multivariable multilevel logistic regressions were
\end{abstract}

Karger@karger.com www.karger.com/mpp

Karger $\stackrel{\text { ' }}{5}$

GOPEN ACCESS
(C) 2022 The Author(s)

Published by S. Karger AG, Basel

This is an Open Access article licensed under the Creative Commons Attribution-NonCommercial-4.0 International License (CC BY-NC) (http://www.karger.com/Services/OpenAccessLicense), applicable to the online version of the article only. Usage and distribution for commercial purposes requires written permission. used to fit regression models for the binary primary outcome of hospital mortality and the secondary outcome intensive care unit (ICU) mortality with hospital unit as random effect. Optimal cutoffs were calculated using the Youden index. $\boldsymbol{R e}$ sults: Patients with high RDW were more often older than 65 years $(57 \%$ vs. $50 \% ; p<0.001)$ and had higher Acute Physiology and Chronic Health Evaluation (APACHE) IV scores (69vs. 60 pts.; $p<0.001$ ). Both hospital (adjusted odds ratios [aOR] 1.18; 95\% Cl: 1.16-1.20; $p<0.001$ ) and ICU mortality (aOR $1.16 ; 95 \% \mathrm{Cl}: 1.14-1.18 ; p<0.001)$ were associated with RDW as a continuous variable. Patients with high RDW had a higher hospital mortality ( 20 vs. $9 \%$; aOR $2.63 ; 95 \%$ Cl: $2.38-2.90$; $p<0.001)$. This finding persisted after multivariable adjustment (aOR 2.14; 95\% Cl: 1.93-2.37; $p<0.001$ ) in a multilevel 
logistic regression analysis. The optimal RDW cutoff for the prediction of hospital mortality was $16 \%$. Conclusion: We found an association of RDW with mortality in septic patients and propose an optimal cutoff value for risk stratification. In a combined model with lactate, RDW shows equivalent diagnostic performance to Sequential Organ Failure Assessment (SOFA) score and APACHE IV score.

(C) 2022 The Author(s).

Published by S. Karger AG, Basel

\section{Introduction}

Sepsis is characterized by an excessive immune response of the body against an infectious pathogen [1]. It is not only associated with high morbidity, but it is also a very common entity [2-6]. Innovative new treatment concepts as well as early diagnosis and the implementation of evidence-based treatment algorithms are possibilities to improve the outcomes of affected patients [7, 8]. New reliable, easily available, and pragmatic risk parameters could help to further optimize the management of septic patients via early identification of high-risk patients and subgroups [9]. This might help to guide timely and aggressive therapy on the one hand, and to adequately allocate intensive care resources on the other hand. Resource allocation might be of special interest in an ongoing pandemic with limited intensive care resources.

The red cell distribution width (RDW) is calculated on the basis of the mean corpuscular volume of erythrocytes and its standard deviation. It can be altered in numerous acute and chronic diseases. Different authors showed an association of high RDW with adverse outcomes in patients with acute heart failure [10], pancreatitis [11], pulmonary embolism [12], acute renal failure [13], stroke [14], influenza [15], and sepsis [16-18]. RDW is a marker of anisocytosis reflecting the heterogeneity of erythrocyte volume [19].

Previous studies examining the association of RDW with mortality in septic patients were often conducted in small single-center cohorts [16]. While almost all blood analyzers calculate RDW and the formula for its calculation should be consistent, devices from different manufacturers are probably different in terms of the technology used. Therefore, the RDW limits of 1 patient population may be slightly different from those of another population when various brands of analyzers are used. Therefore, we sought to compensate for small variances by conducting a large multicenter study with more than 16,000 septic patients and also to find an optimal cutoff value for RDW in septic patients.

\section{Methods}

Study Subjects and Ethics

All critically ill patients admitted to an intensive care unit (ICU) with a principal diagnosis of sepsis and a documented RDW were included in this analysis from the eICU Collaborative Research Database, which is a multicenter ICU database, including over 200,000 deidentified admissions of 335 ICUs from 208 hospitals across the USA in 2014 and 2015 [20, 21]. An Institutional Review Board (IRB; Massachusetts Institute of Technology, Cambridge, MA, USA) approval exists; therefore, no further approval was required. The reidentification risk was certified as meeting safe harbor standards (Privacert, Cambridge, MA, USA; HIPAA Certification No. 1031219-2). Septic patients in this study were identified as previously described [22]. We included only the first admission of each patient and included all patients with sepsis and a documented RDW value within the first $24 \mathrm{~h}$ after admission.

\section{Statistical Analysis}

We extracted baseline characteristics on day 1 if not stated otherwise explicitly. We expressed continuous data points as median \pm interquartile range and differences between independent groups using the Mann-Whitney U test. Categorical data are given as numbers (percentage), and the $\chi^{2}$ test was used to calculate univariate differences between groups.

The primary exposure was the RDW, both as a continuous variable and dichotomized into low $(\leq 15 \%)$ and high $(>15 \%)$ RDW (according to the empirical cutoff used in most hospitals and laboratories). The primary outcome of our analysis was hospital mortality. As secondary outcomes, we evaluated the frequencies of mechanical ventilation, vasopressor use, and acute kidney injury within $48 \mathrm{~h}$ based on the serum creatinine and according to Kidney Disease Improving Global Outcomes (KDIGO) guidelines, length of stay (in hours), complicated stay defined as length of stay $>7$ days, and ICU mortality. We used a multilevel logistic regression analysis to fit a regression model with the hospital unit as a random effect and RDW as a fixed effect, as well as two multivariable models adjusting for (1) Acute Physiology and Chronic Health Evaluation (APACHE) IV and (2) Sequential Organ Failure Assessment (SOFA) scores. We calculated adjusted odds ratios (aOR) with respective 95\% confidence intervals (95\% CI).

We performed sensitivity analyses, stratifying patients with and without septic shock (according to Sepsis-3 criteria), mechanical ventilation, vasopressor use, and for gender, age above and below 65 years, non-Caucasian and Caucasian patients, and pulmonary versus nonpulmonary focus, as well as patients stratified for hemoglobin and mean cellular volume (MCV). For the prediction model, which combined RDW and lactate concentration at admission, we fitted regression coefficients with logistic regression analysis for hospital mortality. All tests were two-sided, and a $p$ value of $<0.05$ was considered statistically significant. We used Stata/IC 16.1 (StataCorp. 2019, Stata Statistical Software: Release 16, College Station, TX, USA: StataCorp. LLC) for all statistical analyses. 
Table 1. Baseline characteristics in the total cohort stratified for low $(\leq 15 \%)$ and high $(>15 \%)$ red cell distribution width (RDW)
Table 2. Outcomes of the total cohort in uni- and multivariable multilevel logistic regression analyses

\begin{tabular}{lllc}
\hline Characteristic & $\begin{array}{l}\text { RDW } \leq 15 \% \\
(n=7,129)\end{array}$ & $\begin{array}{l}\text { RDW }>15 \% \\
(n=9,294)\end{array}$ & $p$ value \\
\hline Age, years, median (IQR) & $66(54-77)$ & $68(58-79)$ & $<0.001$ \\
Age $>65$ years, \% (n) & $50(3,584)$ & $57(5,334)$ & $<0.001$ \\
Male sex, \% ( $n)$ & $52(3,693)$ & $50(4,634)$ & 0.013 \\
BMI, kg/m², median (IQR) & $27(23-32)$ & $27(23-33)$ & 0.34 \\
Caucasian, \% ( $n)$ & $81(5,769)$ & $77(7,178)$ & $<0.001$ \\
Septic shock, \% $(n)$ & $14(630)$ & $21(1,232)$ & $<0.001$ \\
Cutaneous focus, \% (n) & $8(603)$ & $9(805)$ & 0.64 \\
Pulmonary focus, \% (n) & $39(2,812)$ & $37(3,412)$ & $<0.001$ \\
Gl focus, \% $(n)$ & $12(845)$ & $12(1,144)$ & 0.37 \\
UTI focus, \% $(n)$ & $24(1,721)$ & $23(2,168)$ & 0.22 \\
Gynecologic focus, \% (n) & $0.3(27)$ & $0.2(20)$ & 0.052 \\
Other focus, \% ( $n)$ & $6(394)$ & $7(636)$ & $<0.001$ \\
Unknown focus, \% (n) & $10(727)$ & $12(1,109)$ & $<0.001$ \\
SOFA score, points, median (IQR) & $5(3-7)$ & $6(4-9)$ & $<0.001$ \\
APACHE IV score, points, median (IQR) & $60(46-76)$ & $69(53-87)$ & $<0.001$ \\
Hemoglobin, g/dL, median (IQR) & $11(10-12)$ & $10(8-11)$ & $<0.001$ \\
MCV, fL, median (IQR) & $92(88-96)$ & $90(85-95)$ & $<0.001$ \\
MCH, pg, median (IQR) & $30(29-32)$ & $29(27-31)$ & $<0.001$ \\
MCHC, g/dL, median (IQR) & $33(32-34)$ & $32(31-33)$ & $<0.001$ \\
White blood count, G/L, median (IQR) & $13(9-18)$ & $13(8-19)$ & 0.52 \\
Platelet count, G/L, median (IQR) & $182(133-243)$ & $183(119-262)$ & 0.98 \\
Creatinine, mg/dL, median (IQR) & $1(1-2)$ & $1(1-3)$ & $<0.001$ \\
Lactate, mmol/L, median (IQR) & $2(1-3)$ & $2(1-4)$ & $<0.001$ \\
Baseline lactate $>2.0$ mmol/L, \% (n) & $39(1,758)$ & $44(2,669)$ & $<0.001$ \\
\hline
\end{tabular}

APACHE IV, acute physiology and chronic health evaluation IV; BMI, body mass index; $\mathrm{Gl}$, gastrointestinal; $\mathrm{MCH}$, mean cellular hemoglobin; $\mathrm{MCHC}$, mean cellular hemoglobin concentration; MCV, mean cellular volume; SOFA, sequential organ failure assessment; UTI, urinary tract infection.

\begin{tabular}{llllc}
\hline Characteristic & $\begin{array}{l}\text { RDW } \leq 15 \% \\
(n=7,129)\end{array}$ & $\begin{array}{l}\text { RDW }>15 \% \\
(n=9,294)\end{array}$ & $p$ value & $\begin{array}{l}\text { Adjusted } \\
p \text { value }\end{array}$ \\
\hline Mechanical ventilation, $\%(n)$ & $20(1,411)$ & $23(2,109)$ & $<0.001$ & $<0.001$ \\
Vasopressor use, $\%(n)$ & $26(1,859)$ & $35(3,207)$ & $<0.001$ & $<0.001$ \\
LOS $>7$ days, $\%(n)$ & $12(828)$ & $13(1,251)$ & $<0.001$ & 0.39 \\
AKI, $\%(n)$ & $5(321)$ & $7(583)$ & $<0.001$ & 0.001 \\
Hospital mortality, $\%(n)$ & $9(609)$ & $20(1,853)$ & $<0.001$ & $<0.001$ \\
ICU mortality, $\%(n)$ & $5(382)$ & $12(1,153)$ & $<0.001$ & $<0.001$ \\
\hline
\end{tabular}

AKI, acute kidney injury; APACHE IV, acute physiology and chronic health evaluation IV; ICU, intensive care unit; LOS, length of stay; RDW, red cell distribution width. ${ }^{*}$ Multilevel logistic regression with the hospital unit as random effect and RDW as fixed effect, adjusted for APACHE IV. ${ }^{* *}$ Multilevel linear regression with the hospital unit as random effect and RDW as fixed effect, adjusted for APACHE IV.

\section{Results}

\section{Total Cohort}

In total, 16,423 septic patients were included in this analysis, 7,129 with RDW $\leq 15 \%$ and 9,294 with RDW
$>15 \%$. Patients with RDW $>15 \%$ were more often older than 65 years ( $57 \%$ vs. $50 \% ; p<0.001)$, evidenced higher SOFA ( 6 pts. vs. 5 pts.; $p<0.001)$ and APACHE IV (69 pts. vs. 60 pts.; $p<0.001)$ scores, and suffered from septic shock more often $(21 \%$ vs. $14 \% ; p<0.001)$ (Table 1$)$. 


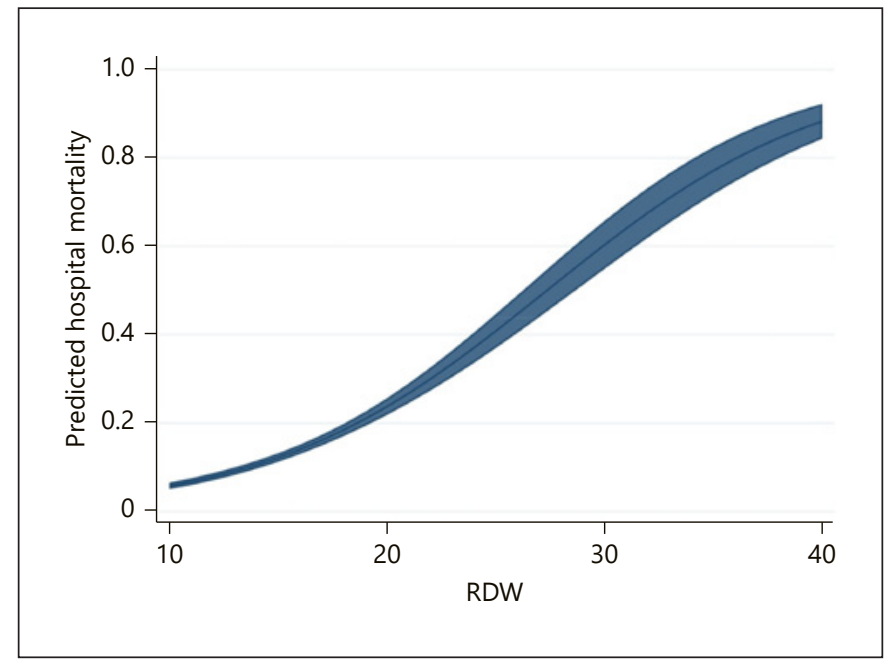

Fig. 1. Correlation of predicted hospital mortality based on RDW values (in \%).

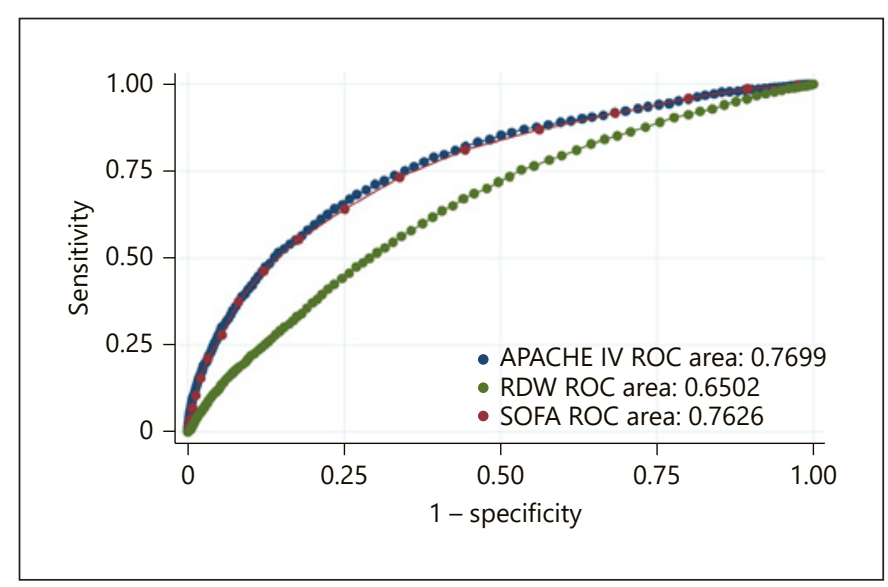

Fig. 2. ROC curves for SOFA score, APACHE IV score, and RDW. APACHE IV, acute physiology and chronic health evaluation; RDW, red cell distribution width; SOFA, sequential organ failure assessment; ROC, receiver-operating characteristic.

Patients with RDW $>15 \%$ received mechanical ventilation ( $23 \%$ vs. $20 \%)$ and vasopressors (35\% vs. $26 \%)$ more often and suffered from acute kidney injury to a greater extent (7\% vs. 5\%). These findings persisted after adjustment for APACHE IV score (see Table 2). A pulmonary focus was more often present in patients with RDW $<15 \%$ (39\% vs. $37 \%$; $p<0.001$ ). No differences were found for other specific infectious foci.

RDW as a continuous variable was associated with hospital mortality (aOR 1.18; 95\% CI: 1.16-1.20; $p<0.001$; see Fig. 1) and remained so after multivariable adjustment for
APACHE IV (aOR 1.15; 95\% CI: $1.13-1.17 ; p<0.001)$ and SOFA scores (aOR 1.13; 95\% CI: 1.12-1.15; $p<0.001$ ) and age (aOR 1.18; 95\% CI: 1.16-1.20; $p<0.001$ ). Receiveroperating characteristic curves of SOFA and APACHE IV scores and RDW are shown in Figure 2. Further, RDW was associated with ICU mortality (aOR 1.16; 95\% CI: $1.14-1.18 ; p<0.001$ ), whereas this finding remained statistically significant after adjustment for both APACHE IV (aOR 1.12; 95\% CI: $1.10-1.14 ; p<0.001)$ and SOFA scores (aOR 1.10; 95\% CI: 1.08-1.12; $p<0.001)$ and age (aOR 1.16; 95\% CI: 1.14-1.18; $p<0.001$ ).

Patients with RDW $>15 \%$ exhibited significantly higher hospital $(20 \%$ vs. $9 \%$; $p<0.001)$ and ICU mortality $(12 \%$ vs. $5 \%$; $p<0.001)$. Both associations remained statistically significant after adjustment for APACHE IV and SOFA scores (hospital mortality: aOR 2.12; 95\% CI: 1.92-2.35; $p<0.001$; ICU mortality: aOR 1.82; 95\% CI: $1.62-2.05 ; p<0.001)$ and age.

In the sensitivity analyses, higher mortality rates were observed in patients in all evaluated subgroups with RDW $>15 \%$ (Fig. 3). The AUC for RDW was 0.65 (95\% CI: 0.64-0.66) and therefore lower than for APACHE IV (AUC 0.77) and SOFA scores (AUC 0.76). However, after combining the RDW with baseline lactate in a prediction model, the AUC $(0.75 ; p=0.05)$ was similar compared to both APACHE IV and SOFA scores. The optimal cutoff for RDW for the prediction of hospital mortality was $15.55 \%$.

\section{Discussion}

We found a robust and independent association of RDW with mortality in septic patients. This finding persisted after adjustment for both APACHE IV and SOFA scores, as well as in several sensitivity analyses. Compared with these established intensive care risk parameters, RDW had a significantly lower prognostic capacity. Nevertheless, it achieved similar prognostic performance together with lactate in a simple combined model.

This study, based on our knowledge of the published literature, is the largest study examining the association of RDW with mortality in septic patients, and its study cohort is approximately as large as the pooled cohort in the recent meta-analysis of Zhang et al. [16]. Thus, the results of our study not only confirm the previous smaller analyses in septic patients but also add a number of important aspects to the existing literature.

Furthermore, previous association analyses of RDW and mortality in septic patients were mainly performed 
Fig. 3. Forest plot of the sensitivity analyses, stratifying patients with and without septic shock, mechanical ventilation, vasopressor use, and for gender, age above and below 65 years, non-Caucasian and Caucasian patients, and pulmonary versus nonpulmonary focus, as well as patients stratified for hemoglobin values and MCV. CI, confidence interval; $\mathrm{Hb}$, hemoglobin; MCV, mean cellular volume.

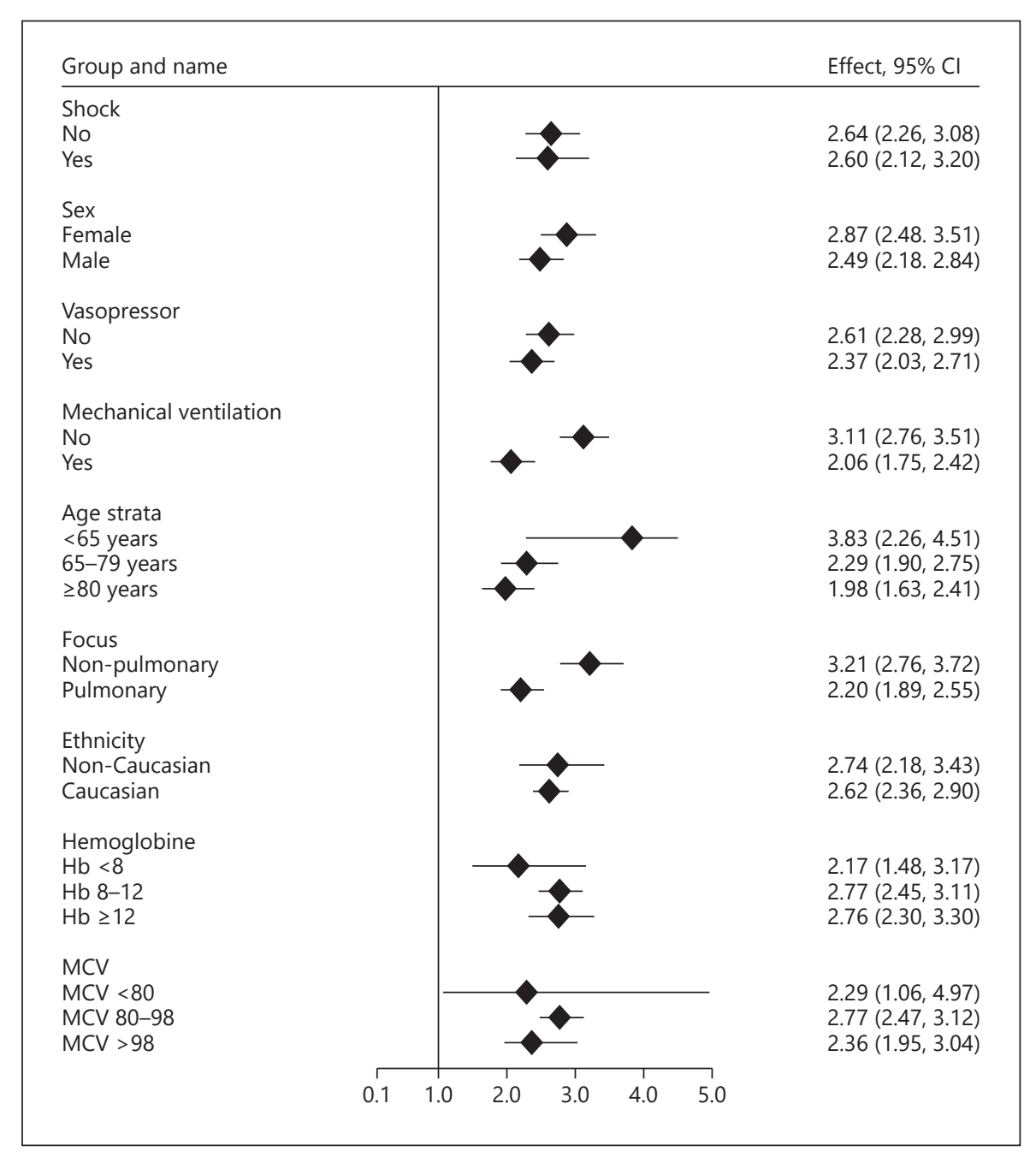

in patients of Asian ethnicity [16], whereas our cohort consists mainly of Caucasian patients. In the sensitivity analysis, we also did not find any evidence of a differential association of RDW with mortality between Caucasian and non-Caucasian patients. Also, RDW was robustly associated with mortality in patients with both sepsis and septic shock, in both sexes and regardless of the infectious focus.

The possible pathophysiological mechanisms underlying the observed robust association of RDW and mortality in septic patients are beyond the scope of this study, and we can therefore only speculate about it based on the existing literature [19]. RDW is a marker of anisocytosis and hence reflects the heterogeneity of erythrocyte volume [19]. It is significantly associated with the abnormal erythropoietin production or effectiveness [23, 24]. Cheng et al. [25] reported an association of RDW with increasing age. In our cohort of septic patients, patients with high RDW were older; however, the association of RDW with mortality remained independent of age.

As RDW is calculated using the MCV, parameters that alter the MCV can also influence the RDW. As for known confounders of MCV such as vitamin B12 and folic acid status, iron status, or the presence of, for example, myelodysplastic disorders, unfortunately we do not have any data. However, in our study, RDW was associated with mortality in patients with both low and high hemoglobin values and also low and high MCV and therefore seems to be independently associated with mortality regardless of the underlying hematological process that led to anisocytosis.

The question of whether RDW is a mere marker for patients at high risk of dying or whether RDW is causally involved in the pathophysiology of acute disease remains open. What is evident is that RDW is associated with numerous factors associated with increased biological age 
and thus risk of death, such as shortened telomere length, chronic low-grade inflammation, renal dysfunction, blood transfusions, nutritional status, and low serum antioxidant concentrations $[17,26,27]$. Interestingly, in a study by Fontana et al. [28] on 122 septic patients, no correlation of RDW with microcirculatory alterations was shown by means of capillary microscopy. Whether there is, for example, a particular value for tailored treatment with antioxidants, anti-inflammatory immunomodulators, or high-caloric diet in patients with increased RDW is beyond our knowledge and should be the subject of future investigation. However, given that erythrocytes are the most abundant cells in the blood, and the essential role of erythrocytes in various acute and chronic diseases is just beginning to be understood, we believe that these associations should at least be considered [29-31]. To what extent intensive care measures such as use of vasopressors, renal replacement therapy, antimicrobial agents, therapeutic hypothermia, and so on influence RDW during the course of the stay cannot be answered by this study, which investigates RDW values directly after ICU admission. Nevertheless, this would be necessary for the evaluation of RDW as a follow-up parameter, which may even indicate a therapy response.

Blood transfusions are a relevant measure in critically ill patients. They also represent a potential confounder in RDW measurements as they increase RDW values [27, 32]. In this database, unfortunately, we have no information regarding transfusions before ICU admission, which would certainly be the most relevant parameter to address for this issue. In their work, Fogagnolo et al. [32] were able to show that RDW is associated with mortality in critically ill patients independently of red blood cell transfusions. Nevertheless, they found higher ideal RDW cutoffs for transfused patients (nontransfused patients $14.3 \%$, transfused patients $15.3 \%)$. Still, we think that due to the large number of patients in our study, this bias would not have significantly affected our results, especially because septic patients frequently receive initial fluid resuscitation with crystalloids, and transfusions often become necessary during the course.

The AUC of RDW with respect to hospital mortality was significantly lower than in SOFA and APACHE IV scores. Yet, in our opinion, RDW has an advantage over SOFA and APACHE IV, as it is significantly easier to determine. RDW can be quickly and easily read from the admission laboratory reports and is also not based on volatile parameters such as blood pressure and heart rate. It could be a simple but relatively reliable parameter for risk stratification of septic patients, possibly even before ad- mission to the ICU in the emergency department. Nevertheless, it should be noted that our study evaluated only ICU patients. Since RDW is independently associated with mortality in septic patients, it could also contribute to the granularity of established risk scores. It would also be conceivable to include RDW in possible new versions of more complex risk scores, or to combine RDW with clinical parameters to allow rapid and even more accurate risk stratification.

Lactate is a well-known parameter for risk stratification of critically ill patients and an indicator of a general "alarm state" in the body. Especially in sepsis, persistent hyperlactatemia despite sufficient fluid resuscitation indicates the presence of septic shock [1]. In particular, persistent hyperlactatemia and severe hyperlactatemia ( $>10 \mathrm{mmol} / \mathrm{L})$ are known indicators of poor outcome [33]. RDW, like lactate, is a dynamic marker and increasing levels during a stay in the ICU are associated with higher mortality [26]. It would certainly be interesting to plan a study that examines the dynamics of both markers in critically ill patients and combines the prognostic significance of persistent hyperlactatemia and increasing RDW. Of note, in our cohort, the combination of RDW with lactate yielded an AUC similar to far more complex prediction tools such as APACHE IV and SOFA scores. In fact, because the combination of lactate and RDW could be easily calculated and displayed in laboratory reports, we consider this tool to be advantageous for clinical practice compared with complex risk scores, which are often difficult to calculate retrospectively in clinical practice.

Limitations of this study include the fact that data on the different blood counting analyzers used were not available. This could have resulted in potential variations in RDW measurement. Also, we have no information regarding transfusions prior to measurement of RDW on ICU admission. This could have influenced RDW in affected patients.

\section{Conclusions}

We demonstrated an association of RDW and mortality in septic patients. Together with lactate, it shows equivalent diagnostic performance to complex intensive care scores such as SOFA score and APACHE IV score. This model could play a role for rapid, inexpensive, and pragmatic risk stratification in combination with clinical judgment. 


\section{Statement of Ethics}

In accordance with institutional requirements and national legislation, and since this is an analysis of a publicly available, anonymized database, no individual patient consent is required for this study. An approval from the Institutional Review Board (IRB; Massachusetts Institute of Technology, Cambridge, MA, USA) for the creation of the eICU database exists, which is released under the safe harbor provision of the Health Insurance Portability and Accountability Act (HIPAA).

\section{Conflict of Interest Statement}

The authors declare that they have no known competing financial interests or personal relationships that could have influenced the work reported in this paper.

\section{Funding Sources}

This research received no specific grant from any funding agency in the public, commercial, or not-for profit sectors.

\section{Author Contributions}

D.D., R.R., B.M., and B.W. analyzed the data and wrote the first draft of the manuscript. Z.Z., S.W., and V.O. gave guidance and improved the paper.

\section{Data Availability Statement}

All data relevant for this study will be given by the authors upon specific request.

\section{References}

1 Singer M, Deutschman CS, Seymour CW, Shankar-Hari M, Annane D, Bauer M, et al. The third international consensus definitions for sepsis and septic shock (sepsis-3). JAMA. Feb 23 2016;315(8):801-10.

2 Vincent JL, Marshall JC, Namendys-Silva SA, Francois B, Martin-Loeches I, Lipman J, et al. Assessment of the worldwide burden of critical illness: the intensive care over nations (ICON) audit. Lancet Respir Med. May 2014; 2(5):380-6.

3 van Vught LA, Klein Klouwenberg PM, Spitoni C, Scicluna BP, Wiewel MA, Horn J, et al. Incidence, risk factors, and attributable mortality of secondary infections in the intensive care unit after admission for sepsis. JAMA. Apr 12 2016;315(14):1469-79.

4 Sakr Y, Moreira CL, Rhodes A, Ferguson ND, Kleinpell R, Pikkers P, et al. The impact of hospital and ICU organizational factors on outcome in critically ill patients: results from the extended prevalence of infection in Intensive Care study. Crit Care Med. Mar 2015; 43(3):519-26.

5 Prescott HC, Angus DC. Enhancing recovery from sepsis: a review. JAMA. Jan 2 2018; 319(1):62-75.

6 Martin GS, Mannino DM, Moss M. The effect of age on the development and outcome of adult sepsis. Crit Care Med. Jan 2006;34(1): $15-21$.

7 Marik PE. The management of sepsis: science \& fiction. J Thorac Dis. Feb 2020;12(Suppl 1): S1-4.

8 Zimmerman JE, Kramer AA, McNair DS, Malila FM. Acute physiology and chronic health evaluation (APACHE) IV: hospital mortality assessment for today's critically ill patients. Crit Care Med. May 2006;34(5): 1297-310.
9 Wernly B, Mamandipoor B, Baldia P, Jung C, Osmani V. Machine learning predicts mortality in septic patients using only routinely available ABG variables: a multi-centre evaluation. Int J Med Inform. Jan 2021;145:104312.

10 Salvatori M, Formiga F, Moreno-Gonzalez R, Chivite D, Migone De Amicis M, Cappellini $\mathrm{MD}$, et al. Red blood cell distribution width as a prognostic factor of mortality in elderly patients firstly hospitalized due to heart failure. Kardiol Pol. Jun 25 2019;77(6):632-8.

11 Zhang T, Liu H, Wang D, Zong P, Guo C, Wang $F$, et al. Predicting the severity of acute pancreatitis with red cell distribution width at early admission stage. Shock. May 2018;49(5): 551-5.

12 Yazici S, Kiris T, Sadik Ceylan U, Terzi S, Uzun AO, Emre A, et al. Relation between dynamic change of red cell distribution width and 30-day mortality in patients with acute pulmonary embolism. Clin Respir J. Mar 2018;12(3):953-60.

13 Wang B, Lu H, Gong Y, Ying B, Cheng B. The association between red blood cell distribution width and mortality in critically ill patients with acute kidney injury. Biomed Res Int. 2018;2018:9658216.

14 Li B, Liu S, Liu X, Fang J, Zhuang W. Association between red cell distribution width level and risk of stroke: a systematic review and meta-analysis of prospective studies. Medicine. Apr 2020;99(16):e19691.

15 Topaz G, Kitay-Cohen Y, Peled L, Gharra W, Kaminer K, Eitan M, et al. The association between red cell distribution width and poor outcomes in hospitalized patients with influenza. J Crit Care. Oct 2017;41:166-9.

16 Zhang L, Yu CH, Guo KP, Huang CZ, Mo LY. Prognostic role of red blood cell distribution width in patients with sepsis: a systematic review and meta-analysis. BMC Immunol. Jul 6 2020;21(1):40.
17 Hu ZD, Lippi G, Montagnana M. Diagnostic and prognostic value of red blood cell distribution width in sepsis: a narrative review. Clin Biochem. Mar 2020;77:1-6.

18 Wang AY, Ma HP, Kao WF, Tsai SH, Chang CK. Red blood cell distribution width is associated with mortality in elderly patients with sepsis. Am J Emerg Med. Jun 2018;36(6):94953.

19 Salvagno GL, Sanchis-Gomar F, Picanza A Lippi G. Red blood cell distribution width: a simple parameter with multiple clinical applications. Crit Rev Clin Lab Sci. 2015;52(2):86105.

20 Pollard TJ, Johnson AEW, Raffa JD, Celi LA, Mark RG, Badawi O. The eICU collaborative research database, a freely available multicenter database for critical care research. Sci Data. Sep 11 2018;5:180178.

21 O'Halloran HM, Kwong K, Veldhoen RA, Maslove DM. Characterizing the patients, hospitals, and data quality of the eICU collaborative research database. Crit Care Med. Dec 2020;48(12):1737-43.

22 Angus DC, Linde-Zwirble WT, Lidicker J, Clermont G, Carcillo J, Pinsky MR. Epidemiology of severe sepsis in the United States: analysis of incidence, outcome, and associated costs of care. Crit Care Med. Jul 2001;29(7): 1303-10.

23 Kario K, Matsuo T, Nakao K, Yamaguchi N. The correlation between red cell distribution width and serum erythropoietin titres. Clin Lab Haematol. 1991;13(2):222-3.

24 Afsar B, Saglam M, Yuceturk C, Agca E. The relationship between red cell distribution width with erythropoietin resistance in iron replete hemodialysis patients. Eur J Intern Med. Apr 2013;24(3):e25-9. 
25 Cheng CK, Chan J, Cembrowski GS, van Assendelft OW. Complete blood count reference interval diagrams derived from NHANES III: stratification by age, sex, and race. Lab Hematol. 2004;10(1):42-53.

26 Kim CH, Park JT, Kim EJ, Han JH, Han JS, Choi JY, et al. An increase in red blood cell distribution width from baseline predicts mortality in patients with severe sepsis or septic shock. Crit Care. Dec 9 2013;17(6):R282.

27 Spadaro S, Taccone FS, Fogagnolo A, Franchi F, Scolletta S, Ragazzi R, et al. The effects of blood transfusion on red blood cell distribution width in critically ill patients: a pilot study. Transfusion. Aug 2018;58(8):1863-9.
28 Fontana V, Spadaro S, Bond O, Cavicchi FZ, Annoni F, Katia D, et al. No relationship between red blood cell distribution width and microcirculatory alterations in septic patients. Clin Hemorheol Microcirc. 2017; 66(2):131-41.

29 Mahdi A, Tengbom J, Alvarsson M, Wernly B, Zhou Z, Pernow J. Red blood cell peroxynitrite causes endothelial dysfunction in type 2 diabetes mellitus via arginase. Cells. Jul 16 2020;9(7):1712.

30 Pernow J, Mahdi A, Yang J, Zhou Z. Red blood cell dysfunction: a new player in cardiovascular disease. Cardiovasc Res. Sep 1 2019; 115(11):1596-605.
31 Zhou Z, Mahdi A, Tratsiakovich Y, Zahoran S, Kövamees O, Nordin F, et al. Erythrocytes from patients with type 2 diabetes induce endothelial dysfunction via arginase I. J Am Coll Cardiol. Aug 14 2018;72(7):769-80.

32 Fogagnolo A, Spadaro S, Taccone FS, Ragazzi R, Romanello A, Fanni A, et al. The prognostic role of red blood cell distribution width in transfused and non-transfused critically ill patients. Minerva Anestesiol. Nov 2019; 85(11):1159-67.

33 Haas SA, Lange T, Saugel B, Petzoldt M, Fuhrmann V, Metschke M, et al. Severe hyperlactatemia, lactate clearance and mortality in unselected critically ill patients. Intensive Care Med. Feb 2016;42(2):202-10. 